\title{
Polymorphisms in DENND1B gene are associated with asthma and atopy phenotypes in Brazilian children
}

\author{
Bianca S.D. Fiuza ${ }^{a}$, Milca de J. Silva ${ }^{\mathrm{a}}$, Neuza M. Alcântara-Neves ${ }^{\mathrm{a}}$, Maurício L. Barreto ${ }^{\mathrm{b}}$, \\ Ryan dos S. Costa ${ }^{a}$, Camila A. Figueiredo ${ }^{a, *}$ \\ a Departamento de Biorregulação, Laboratório de Imunofarmacologia e Biologia Molecular, Universidade Federal da Bahia (ICS), Bahia, Brazil \\ b Fundação Oswaldo Cruz, Salvador, Bahia, Brazil
}

\section{A R T I C L E I N F O}

\section{Keywords:}

Asthma

Atopy

Polymorphism

DENND1B

\begin{abstract}
A B S T R A C T
Asthma is a heterogeneous disease associated with a complex basis involving environmental factors and individual variabilities. The DENN Domain Containing 1B (DENND1B) gene has an important role on T cell receptor (TCR) down-regulation on Th2 cells and studies have shown that mutations or loss of this factor can be associated with increased Th2 responses and asthma. The aim of this work is to evaluate the association of polymorphisms in the DENND1B with asthma and allergy markers phenotypes in Brazilian children. Genotyping was performed using a commercial panel from Illumina (2.5 Human Omni bead chip) in 1309 participants of SCAALA (Social Change, Asthma, Allergy in Latin American) program. Logistic regressions for asthma and atopy markers were performed using PLINK software 1.9. The analyzes were adjusted for sex, age, helminth infections and ancestry markers. The DENND1B gene was associated with different phenotypes such as severe asthma and atopic markers (specific IgE production, skin prick test and IL-13 production). Among the 166 SNPs analyzed, 72 were associated with asthma and/or allergy markers. In conclusion, polymorphisms in the DENND1B are significantly associated with development of asthma and atopy and these polymorphisms can influence DENND1B expression and consequently, asthma.
\end{abstract}

\section{Introduction}

Asthma is a complex and heterogeneous disease characterized by chronic airway inflammation, bronchial hyper responsiveness, remodeling and airflow obstruction spontaneously reversible or with treatment, it is manifested by recurrent episodes of wheezing, dyspnea, chest tightness and cough (Barreto et al., 2014; Troy et al., 2016). About 334 million people in the world suffer from asthma (Asher and Pearce, 2014) and an estimated 100 million asthma cases are estimated to increase by 2025 worldwide (Barreto et al., 2014). The prevalence of asthma has been growing steadily throughout the world, affecting mainly children (GINA, 2016).

There are several factors suggested to explain the increase in the prevalence of asthma, including environmental, nutritional, economic and psychosocial aspects (Barreto et al., 2014). The classic risk factors are: family history of allergic diseases, sensitization to environmental allergens (including aeroallergens), endotoxins exposure, fungi and viral respiratory infections in early life; however, such factors do not fully explain the establishment and maintenance of the disease, and other factors have been associated with this disease such as being male, low birth weight, maternal or domestic smoking, secondary exposure to pollution in large cities, climatic changes, exposure to chemical irritants, physical exercise and psychosocial factors (Coelho et al., 2016).

Th2 cells are the most studied cell phenotype in asthma, however evidence shows that other helper T cell lines, such as Th1, Th17 and Tregs, act in different ways in the immunopathology of asthma, because of that it has been suggested that asthmatics can be divided into different subgroups (Malmhäll et al., 2012), being typically classified as atopic and non-atopic asthma. Atopic asthma is characterized by Th2type TCD4 + cells, which secrete cytokines such as IL-4, IL-5 and IL-13, and granulocyte-macrophage colony-stimulating factor (GM-CSF), which in turn leads to the IgE, mast cell and eosinophilic responses that

\footnotetext{
Abbreviations: BDCA, markers for distinct subsets of dendritic cells in human peripheral blood; CI, confidence interval; DENN, differentially expressed in normal and neoplastic cells

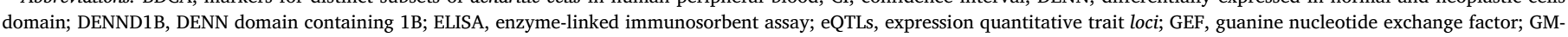

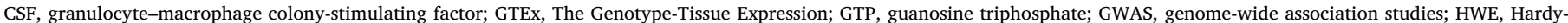

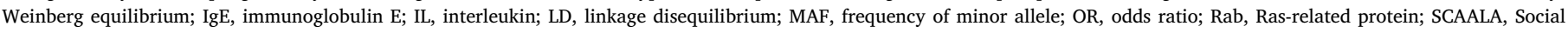
Change, Asthma, Allergy in Latin American; SNP, single nucleotide polymorphism; TCR, T cell receptor; Th cells, T helper cells; TF, transcription factor; Tregs, regulatory T cell

* Corresponding author.

E-mail addresses: camilavf@ufba.br, cavfigueiredo@gmail.com (C.A. Figueiredo).
} 

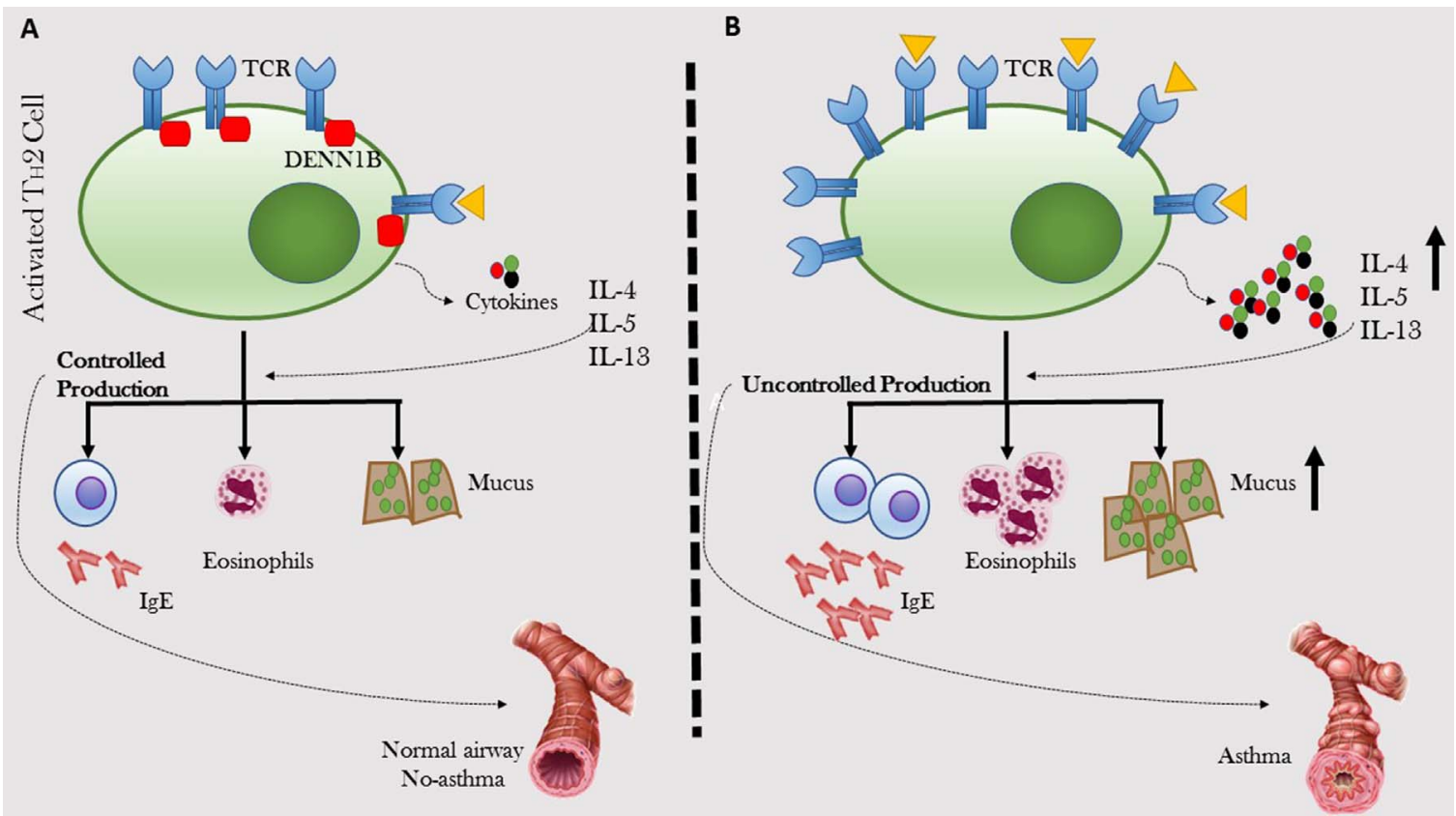

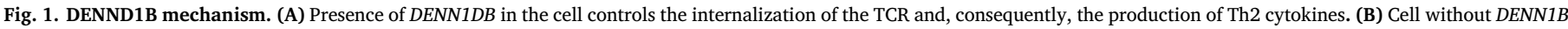
has exacerbated production of cytokines, leading to Th2 asthma.

are characteristic of allergic asthma (Holgate et al., 2015). Asthmatic patients that do not manifest atopy (non-atopic asthma) are called intrinsic asthmatics and they normally show negative skin tests and there is no clinical or family history of allergy. Additionally, lack of specific IgE antibodies directed against common allergens (Humbert et al., 1999).

The heterogeneous condition of asthma arises from different causes associated with a complex genetic basis involving environmental factors and individual variabilities (Kabesch et al., 2007). A number of studies on candidate genes have been conducted in recent years in an attempt to understand the genetic mechanisms of asthma (Costa et al., 2015). Several candidate genes, at different levels, have been associated with different asthma phenotypes, fifty-three genes have been identified and replicated in more than one study, and 20 genes have been replicated in more than 10 separated investigations (Vercelli, 2008).

Genetic polymorphisms, along with environmental factors, play a significant role in asthma pathogenesis, and therefore interest in asthma genetics has grown over the last two decades (Bazzi et al., 2011). The main type of genetic variation observed in individuals is single nucleotide polymorphism (SNP), which can alter the structure, function, levels and expression of proteins (Hawkins et al., 2006).

The differentially expressed in normal and neoplastic cells domain (DENN) interacts with members of the Rab family of small GTPases and has enzymatic function as a guanine nucleotide exchange factor (GEF) for Rab, being able to activate monomeric GTPases by stimulating the release of guanosine diphosphate (GDP) and allowing binding to guanosine triphosphate (GTP) (Yang et al., 2016). In addition, it controls proteins that have been implicated in various cellular functions, including the recycling receptor, synaptic vesicle endocytosis and autophagy (Cherfils and Zeghouf, 2013; Yang et al., 2016).

There are 18 human proteins containing the DENN domain and their alternative splicing variants. Mutations (coding for a different amino acid), chromosomal translocations and altered levels of expression have been described in association with neurological disorders, ocular disorders and cancers (Yang et al., 2016).

The DENND1B gene is located on chromosome 1, region 1q31.3 and is expressed in a subgroup of dendritic cells (BDCA3 + and BDCA4+) and natural killer cells and also in activated $\mathrm{T}$ cells (Sleiman et al., 2010). In addition, it is GEF for Rab35, a key endocytic recycling regulator and important for negative modulation of the $\mathrm{T}$ cell receptor (TCR) in Th2 cells (Yang et al., 2016).

In 2016 Yang et al. proposed the following mechanism for DENND1B in mice: in normal conditions, that is, a cell with DENND1B expressing, this gene will control cytokine production of Th2 cells by modulating the duration of $\mathrm{T}$ cell receptor (TCR), when stimulated by an antigen, with a continuous process of endocytosis and recycling via the endosomes. After exposure to a certain antigen, TCRs molecules in activated $\mathrm{T}$ cells are targeted to the immunological synapse but are quickly, within $5-10 \mathrm{~min}$, targeted to endosomes for degradation or recycling (Fig. 1A). In DENND1B-deficient $\mathrm{T}$ cells, TCR internalization is significantly slowed down, leading to prolonged TCR signaling that could increase production of effector cytokines (IL-4, IL-5, IL-13) involved in the pathology of atopic asthma (Godar and Lambrecht, 2016) (Fig. 1B). In this way, mutations or loss of this factor may be associated with increased Th2 responses and asthma (Yang et al., 2016).

The first study about DENND1B was published by Sleiman et al. (2010), a genome-wide association study (GWAS), which identified a locus containing DENND1B on chromosome 1q31.3 significantly associated with susceptibility to asthma in patients of European ancestry.

The present project is a candidate gene, replicated in some populations of the world, but not yet explored in the Brazilian population. Impact of knowing this susceptibility can increase knowledge about the pathophysiology of asthma and the possibility of personalizing the treatment of patients based on genetic information.

No study with polymorphism in the DENND1B gene has been conducted so far in Latin America. In this way, our aim was to evaluate the association of polymorphisms in the gene DENND1B with atopy and asthma phenotypes in Brazilian children.

\section{Material and methods}

\subsection{Study population}

The present study was conducted with 1309 children who had participated in the SCAALA (Social Change, Asthma, Allergy in Latin American) program and lived in the city of Salvador with a population of approximately 2.9 million habitants (IBGE, 2016). The prevalence of wheezing in the last 12 months in school children aged 12-13 years in 
Salvador is approximately $27.1 \%$ (Rodrigues et al., 2008). This population has already been described in other works of the group (Barreto et al., 2006; Rodrigues et al., 2008). The children were recruited in childhood to participate in a prospective study that aimed to measure the impact of a sanitation program in the city of Salvador on child morbidity (Barreto et al., 2007). Data were collected from children born between 1994 and 2001, who lived in sentinel neighborhoods of the city. Standardized questionnaires were applied to the legal guardian of each child between 1997 and 2003 to collect data on demographic and social variables, as well as on the domestic environment (Figueiredo et al., 2010). The children were interviewed again in 2005 and the same demographic and social variables were collected to obtain stool and blood samples for the laboratory tests and isolation of the genetic material. This work has the approval of the National Research Ethics Committee 120.616 and free informed consent was appropriately obtained from the legal guardian of each child.

\subsection{Variables and markers used in the association study}

Asthma symptoms were defined using a Portuguese adapted phase II International Study of Asthma and Allergies in Childhood (ISAAC) questionnaire and the children were classified as asthmatic when the parents reported wheezing in the previous 12 months and at least one of the following situations: diagnosis of asthma by a doctor at any time in their lives; wheezing with exercise in the last 12 months; four or more episodes of wheezing in the last 12 months; and waking up at night due to wheezing episodes in the last 12 months (Costa et al., 2015; Figueiredo et al., 2013). We defined wheeze phenotypes (atopic and nonatopic by a positive IgE result $(>0.70 \mathrm{kU} / \mathrm{L})$ or skin prick test above $3 \mathrm{~mm}$ of diameter.

Severe asthma was considered cases that, besides mentioning wheezing in the last 12 months, had one or more affirmative answers to the following questions: inability to speak at least two complete words during a wheezing crisis, number of attacks greater than 12 in the last year, number of nocturnal awakenings as a result of asthma ( $>1$ night per week) (Simões et al., 2010).

Determination of specific IgE serum concentrations was performed for mite (Dermatophagoides pteronyssinus and Blomia tropicalis) and for cockroach (Blatella germanica, and Periplaneta americana) by using the ImmunoCAPassay (Phadia Diagnostics $\mathrm{AB}$, Uppsala Sweden). Children with $0.70 \mathrm{kU} / \mathrm{L}$ or greater of specific IgE for any allergen tested were considered to have positive results (Figueiredo et al., 2013).

All individuals were submitted to skin puncture tests for seven common aeroallergens: Dermatophagoides pteronyssinus, Blomia tropicalis, Periplaneta Americana, Blatela germanica, fungi (Aspergillus fumigatus and Penicillium notatum), dog and cat epithelium. Saline and $10 \mathrm{mg} / \mathrm{mL}$ histamine solution were used as negative and positive controls, respectively. After $15 \mathrm{~min}$, the diameter wheal was measured and a mean wheal of at least $3 \mathrm{~mm}$ greater than the negative control was considered positive (Figueiredo et al., 2013).

Venous blood was collected into heparinized tubes and cultured at a dilution of 1:4 in RPMI (Gibco, Auckland, New Zealand) containing $10 \mathrm{mmol} / \mathrm{L}$ glutamine (Sigma-Aldrich, St Louis, Mo) and $100 \mu \mathrm{g} / \mathrm{mL}$ gentamicin (Sigma-Aldrich). The cells were cultured within $6 \mathrm{~h}$ of collection in the presence or not of $10 \mu \mathrm{g} / \mathrm{mL}$ endotoxin-free and were maintained in a humidified environment of $5 \% \mathrm{CO} 2$ at $37^{\circ} \mathrm{C}$ for 5 for detection of IL-13 in the presence of pokeweed mitogen (SigmaAldrich). We determined the optimal time course of cytokine accumulation in whole- blood culture via a standardization process in our own laboratory (Figueiredo et al., 2010).

The IL-13 concentration was measured in whole-blood culture supernatants by using commercially available antibody pairs and recombinant cytokine standards (BD Pharmingen, San Diego, CA), by means of sandwich enzyme-linked immunosorbent assay (ELISA) according to the manufacturer's instructions. Cytokine concentrations were determined by interpolation of standard curves. The detection limits (low/high) for the cytokine was $62.5 / 4000 \mathrm{pg} / \mathrm{mL}$. Responders were defined as those children with cytokine concentrations above the lower detection limits.

\subsection{Genomic DNA extraction and genotyping}

DNA extraction was performed from blood samples according to the protocol of the Gentra Puregene Blood Kit (Quiagen). All samples to be genotyped were standardized at a concentration of $5 \mathrm{ng} / \mu \mathrm{L}$ and stored at $-30{ }^{\circ} \mathrm{C}$ until use.

The genotyping was performed using standardized commercial panel 2.5 HumanOmni Beadchip and currently available from Illumina (www.illumina.com). The DENND1B genetic information were extracted from 197473878 to 197744623 position (NC_000001.10) at the chromosome 1. Most of these SNPs were selected from the 3.1 million genotyped SNPs in the HapMap International Project (http://www. hapmap.org/), which represents the largest international initiative for mapping genomic variability and the pattern of imbalance in the human genome (The International HapMap Consortium, 2007).

\subsection{Data analysis}

For genetic association analysis, each SNPs were subjected to quality control tests that include parameters such as genotype frequency, Hardy-Weinberg equilibrium (HWE $>0.001$ ), population stratification, genotypic allocation, frequency of minor allele (MAF > 0.05), and $p$-value of less than 0.05 were consider to associations statically significant. These parameters were evaluated using the software PLINK (version 1.9) (Purcell et al., 2007). Logistic regressions for asthma and allergy markers (skin tests and IgE production) were performed using the same software, adjusted for sex, age, helminth infections and ancestry markers (Principal components (PC)), were used as covariates to control confounding by population structure (Costa et al., 2015). Empirical p values were generated through a permutational approach for correction for multiple tests and the OR value (odds ratio) was estimated. The analyses were performed using the additive genetic model. Pairwise LD was created with Haploview.

\subsection{In silico analysis}

Information about the function of each SNP was obtained in the National Center for Biotechnology Information (NCBI) (www.ncbi.nlm. nih.gov).

RegulomeDB (http://www.regulomedb.org/) is a database that integrates a collection of SNPs with known regulatory information in the intergenic regions of the human genome, thus allowing functional allocation of regulatory information in any set of variants. It uses a scoring system representing the presence of a variant in a functional location that is likely to result in a functional consequence (Boyle et al., 2012). This score ranges from 1 to 6 and the lower this value, greater the probability of this variant being located in a functional region. The 1a to 1f scores are likely to affect binding and linked to expression of a gene target, the $2 \mathrm{a}$ to $2 \mathrm{c}$ scores are likely to affect binding, the $3 \mathrm{a}$ to $3 \mathrm{~b}$ scores are less likely to affect binding, the 4-6 scores are minimal binding evidence (Boyle et al., 2012) and 7 no information (Supplementary Material - Table A1).

The Genotype-Tissue Expression (GTEx) project of the National Institutes of Health Common Fund aims to establish a resource database and associated tissue bank in which to study the relationship between genetic variation and gene expression and other molecular phenotypes in multiple reference tissues (Lonsdale et al., 2013). This project collected and analyzed multiple human tissues from donors who are also densely genotyped, to assess genetic variation within their genomes. By analyzing global RNA expression within individual tissues and treating the expression levels of genes as quantitative traits, variations in gene expression that are highly correlated with genetic variation can be identified as expression quantitative trait loci, or eQTLs (Lonsdale et al., 2013). 
Table 1

Characteristics of the SCAALA population according to asthma status and variables included in this study.

\begin{tabular}{|c|c|c|c|}
\hline \multirow[t]{2}{*}{ Variables } & \multirow{2}{*}{$\frac{\underline{\text { Non-asthmatic }}}{\mathrm{N}(\%)}$} & \multirow{2}{*}{$\frac{\underline{\text { Asthmatic }}}{\mathrm{N}(\%)}$} & \multirow[t]{2}{*}{$\mathrm{p}$-value } \\
\hline & & & \\
\hline Total & 949 & 360 & \\
\hline \multicolumn{4}{|l|}{ Sex (1198) } \\
\hline Male & $594(64.5)$ & 99 (35.7) & $<0.0001^{\mathrm{a}}$ \\
\hline Female & $327(35.5)$ & $178(64.3)$ & $<0.0001^{\mathrm{a}}$ \\
\hline \multicolumn{4}{|l|}{ Age group (1201) } \\
\hline$\leq 5$ years & $341(36.6)$ & $96(35.7)$ & 0.488 \\
\hline $6-7$ years & $320(34.3)$ & $85(31.6)$ & 0.491 \\
\hline$\geq 8$ years & $271(29.1)$ & $88(32.7)$ & 0.419 \\
\hline \multicolumn{4}{|c|}{$\begin{array}{l}\text { a Positive specific IgE production } \\
\text { (1223) }\end{array}$} \\
\hline At least one allergen & $327(51.6)$ & $134(48.4)$ & $<0.0001^{\mathrm{a}}$ \\
\hline D. pteronyssinus & $179(18.9)$ & $91(32.9)$ & $<0.0001^{\mathrm{a}}$ \\
\hline B. tropicalis & $289(30.5)$ & $129(46.6)$ & $<0.0001^{\mathrm{a}}$ \\
\hline B. germanica & $118(12.5)$ & $47(17.5)$ & 0.054 \\
\hline P. americana & $81(8,6)$ & $31(11.2)$ & 0.182 \\
\hline \multicolumn{4}{|c|}{$\begin{array}{l}{ }^{\mathrm{b}} \text { Positive skin prick test response } \\
\quad(1222)\end{array}$} \\
\hline At least one allergen & $273(28.9)$ & $100(36.1)$ & 0.022 \\
\hline D. pteronyssinus & $132(14.0)$ & $62(22.4)$ & 0.001 \\
\hline B. tropicalis & $193(20.4)$ & $76(27.4)$ & 0.013 \\
\hline B. germanica & $71(7,5)$ & $31(11.2)$ & 0.037 \\
\hline P. americana & $120(12.7)$ & 49(17.7) & 0.034 \\
\hline
\end{tabular}

a $>0.70 \mathrm{kU} / \mathrm{L}$.

b $>3 \mathrm{~mm}$ diameter.

\section{Results}

\subsection{Study population and data collection}

Table 1 summarizes the characteristics of the study population and demonstrate that there is statistical difference between both genders and specific IgE production for at least on allergen, D. pteronyssinus and $B$. tropicalis; existing more males in the non-asthmatic group than females, on the other hand, there are more females than males in the asthmatic group.

\subsection{Description of the DENND1B polymorphisms}

In this study, 166 SNPs were extracted from Illumina 2.5 HumanOmni Beadchip. Among these markers, only one was excluded by of Hardy-Weinberg Equilibrium (HWE) test $\left(\mathrm{p} \leq 10^{-3}\right)$ and 93 SNPs were excluded due to very low minor allele frequency (MAF $<0.05$ ). Thus from 166 SNPs typed, 72 were included in the association analysis. Table 2 presents information from 23 SNPs discussed in the present study, that had SNPs with at least two associations with asthma and/or atopy phenotypes and the other 49 with only one association are showed in the supplementary material (Tables A2 and A3).

\subsection{Association of DENND1B polymorphisms with asthma}

The C allele of rs6691216 (OR 0.76, CI 0.60-0.96) was negatively associated with asthma (Table 3). The A allele of rs1421396 (OR 1.45, CI 1.04-2.00) and the allele C of rs1421389 (OR 1.44, CI 1.04-1.99) were positively associated with asthma (Table 3 ) and they are in perfect linkage disequilibrium (Fig. 2).

\subsection{Association of DENND1B polymorphisms with atopic and non-atopic asthma}

The allele $\mathrm{T}$ of rs16841893 (OR 2.37, CI 1.43-3.94), allele C of rs6694441 (OR 2.37, CI 1.43-3.94), allele G of rs73077640 (OR 2.39, CI 1.51-3.79), allele A of rs57589685 (OR 2.00, CI 1.31-3.05) and
Table 2

Base pairs, allele, Minor allele frequency, Hardy-Weinberg equilibrium, function and RegulomeDB score of DENND1B SNPs.

\begin{tabular}{|c|c|c|c|c|c|}
\hline SNP & $\begin{array}{l}\text { Location } \\
\text { allele }^{\mathrm{a}}\end{array}$ & MAF & HWE & Function $^{\mathrm{b}}$ & $\begin{array}{l}\text { RegulomeDB } \\
\text { Score }\end{array}$ \\
\hline rs73077640 & $\mathrm{G} / \mathrm{A}$ & 0.05 & 0.57 & intron variant & 5 \\
\hline rs6694441 & $\mathrm{C} / \mathrm{T}$ & 0.05 & 0.57 & intron variant & 7 \\
\hline rs17641842 & $\mathrm{G} / \mathrm{T}$ & 0.06 & 0.02 & intron variant & 5 \\
\hline rs57589685 & $\mathrm{A} / \mathrm{G}$ & 0.06 & 0.63 & intron variant & 6 \\
\hline rs10801621 & $\mathrm{G} / \mathrm{A}$ & 0.07 & 0.14 & intron variant & 7 \\
\hline rs6685897 & $\mathrm{C} / \mathrm{T}$ & 0.07 & 0.08 & intron variant & 5 \\
\hline rs73073636 & $\mathrm{A} / \mathrm{G}$ & 0.07 & 0.70 & $\begin{array}{l}\text { nc transcript } \\
\text { variant,utr variant } 3 \\
\text { prime }\end{array}$ & 5 \\
\hline rs981520 & $\mathrm{A} / \mathrm{G}$ & 0.08 & 0.72 & intron variant & 5 \\
\hline rs2147771 & $\mathrm{A} / \mathrm{G}$ & 0.08 & 0.73 & intron variant & 7 \\
\hline rs4427436 & $\mathrm{G} / \mathrm{A}$ & 0.08 & 0.73 & $\begin{array}{l}\text { intron variant,utr } \\
\text { variant } 5 \text { prime }\end{array}$ & 6 \\
\hline rs3902061 & $\mathrm{G} / \mathrm{T}$ & 0.08 & 0.73 & intron variant & 5 \\
\hline rs1421396 & $\mathrm{A} / \mathrm{G}$ & 0.09 & 0.74 & intron variant & 4 \\
\hline rs1421389 & $\mathrm{C} / \mathrm{A}$ & 0.09 & 0.74 & $\begin{array}{l}\text { nc transcript } \\
\text { variant,utr variant } 3 \\
\text { prime }\end{array}$ & 5 \\
\hline rs12354257 & $\mathrm{C} / \mathrm{T}$ & 0.19 & 0.92 & intron variant & 7 \\
\hline rs2884629 & $\mathrm{G} / \mathrm{T}$ & 0.20 & 0.44 & intron variant & 7 \\
\hline rs1998598 & $\mathrm{G} / \mathrm{A}$ & 0.20 & 0.50 & intron variant & 6 \\
\hline rs6691216 & $\mathrm{C} / \mathrm{T}$ & 0.25 & 0.41 & intron variant & $3 a$ \\
\hline rs11799915 & $\mathrm{G} / \mathrm{A}$ & 0.27 & 0.88 & intron variant & 6 \\
\hline rs1573100 & $\mathrm{C} / \mathrm{T}$ & 0.30 & 0.43 & intron variant & 5 \\
\hline rs4915551 & $\mathrm{A} / \mathrm{G}$ & 0.46 & 0.26 & intron variant & 7 \\
\hline rs12066841 & $\mathrm{T} / \mathrm{C}$ & 0.46 & 1 & intron variant & 5 \\
\hline rs16841893 & $\mathrm{T} / \mathrm{C}$ & 0.06 & 0.81 & intron variant & 7 \\
\hline rs17641524 & $\mathrm{T} / \mathrm{C}$ & 0.08 & 0.48 & $\begin{array}{l}\text { intron } \\
\text { variant,synonymous } \\
\text { codon,utr variant } 3 \\
\text { prime }\end{array}$ & $3 a$ \\
\hline
\end{tabular}

\footnotetext{
${ }^{\text {a }}$ First is the alternative allele and the second is the reference allele (A1/A2).

b NCBI National Center for Biotechnology Information. MAF: Minor allele frequency. HWE: Hardy-Weinberg equilibrium.
}

Table 3

Association between SNPs in DENND1B gene and asthma by logistic regression adjusted for sex, age, helminth infections and ancestry.

\begin{tabular}{lllll}
\hline SNP & OR & $95 \%$ CI & P value & Perm \\
\hline rs6691216 (C/T) & 0.76 & $0.60-0.96$ & 0.024 & 0.019 \\
rs1421396 (A/G) & 1.45 & $1.04-2.00$ & 0.025 & 0.021 \\
rs1421389 (C/A) & 1.44 & $1.04-1.99$ & 0.027 & 0.023 \\
\hline
\end{tabular}

SNP: single nucleotide polymorphism; OR: odds ratio; 95\% CI: 95\% confidence interval; Perm: permutational $\mathrm{P}$ value for correction for multiple tests.

allele A of rs73073636 (OR 1.72, CI 1.05-2.83), respectively, were positively associated with non-atopic asthma and the allele $\mathrm{C}$ of rs6685897 (OR 1.62, CI 1.04-2.52) was positively associated with atopic asthma when compared with control (Table 4).

\subsection{Association of DENND1B polymorphisms with asthma severity}

The allele A of rs57589685 (OR 1.92, CI 1.24-2.98), allele A of rs73073636 (OR 1.90, CI 1.28-2.83), allele T of rs16841893 (OR 1.71, CI 1.08-2.73), allele C of rs6694441 (OR 1.67, CI 1.02-2.73) and allele $\mathrm{G}$ of rs73077640 (OR 1.67, CI 1.02-2.73) were positively associated with asthma severity and the allele A rs4915551 (OR 0.68, CI 0.53-0.87) and allele C of rs6691216 (OR 0.62, CI 0.45-0.86) were negatively associated with asthma severity (Table 5).

\subsection{Association of DENND1B polymorphisms with specific IgE serum levels}

The results of the analysis between the DENND1B SNPs and specific IgE serum levels are showed in Table 6. The allele A of rs57589685 (OR 


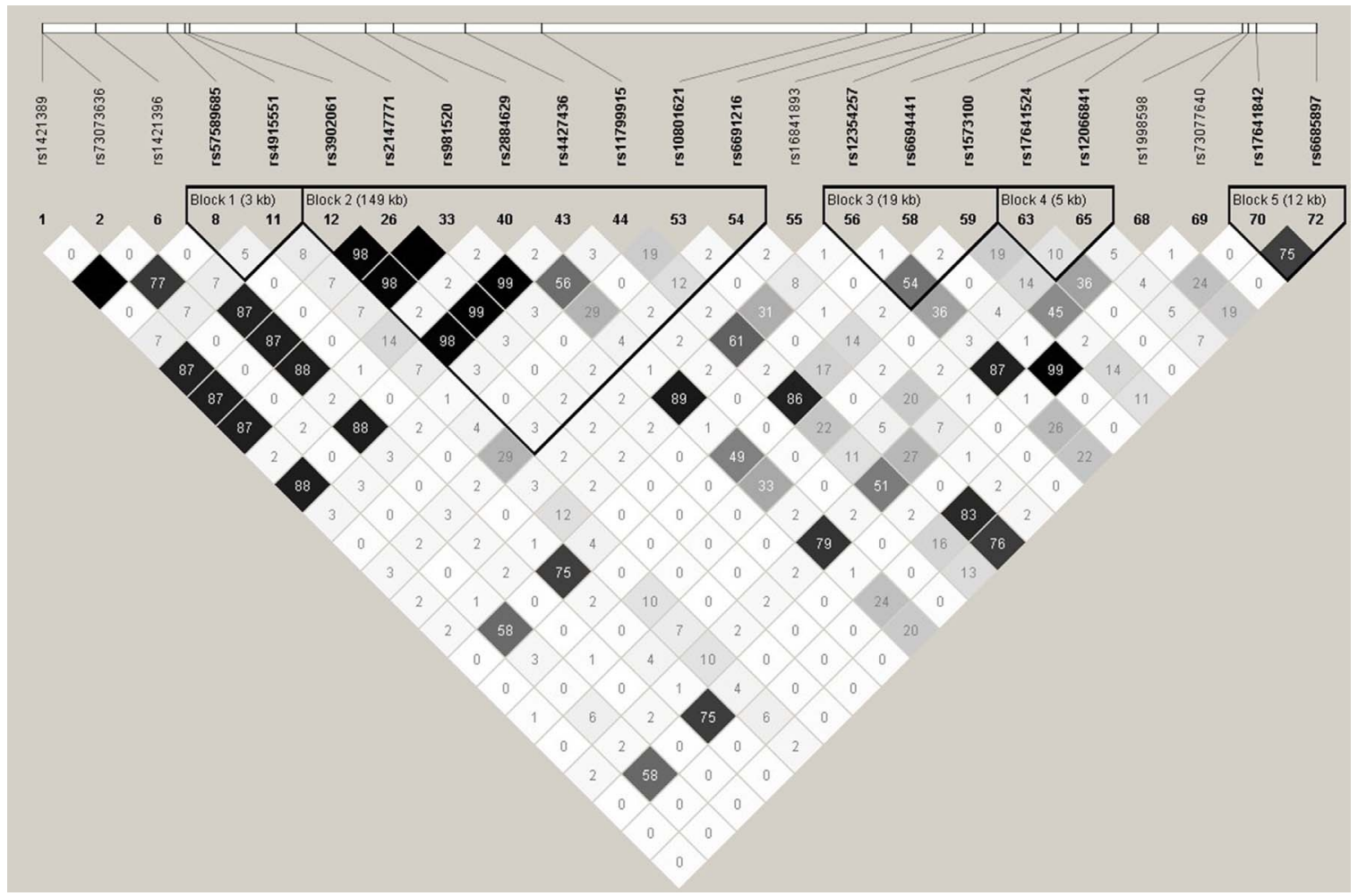

Fig. 2. Pairwise LD within Haploview by using the $\mathrm{R}^{`}$ squared statistic for the DENND1B gene. Intensity of shading indicates the degree of confidence in the $\mathrm{R}^{`}$ value.

Table 4

Association between SNPs in DENND1B gene and atopic and non-atopic asthma by logistic regression adjusted for sex, age, helminth infections and ancestry.

\begin{tabular}{|c|c|c|c|c|}
\hline SNP & OR & $95 \% \mathrm{CI}$ & $P$ value & Perm \\
\hline \multicolumn{5}{|c|}{ Non-atopic Asthma vs Control } \\
\hline rs6694441 (C/T) & 2.37 & $1.43-3.94$ & 0.0007 & 0.0005 \\
\hline $\operatorname{rs} 73077640(\mathrm{G} / \mathrm{A})$ & 2.37 & $1.43-3.94$ & 0.0007 & 0.0005 \\
\hline rs57589685 (A/G) & 2.39 & $1.51-3.79$ & 0.0002 & 0.0001 \\
\hline rs73073636 (A/G) & 2.00 & $1.31-3.05$ & 0.0011 & 0.0010 \\
\hline rs16841893 (T/C) & 1.72 & $1.05-2.83$ & 0.0312 & 0.0302 \\
\hline \multicolumn{5}{|c|}{ Atopic Asthma vs Control } \\
\hline rs6685897 (C/T) & 1.62 & $1.04-2.52$ & 0.0308 & 0.0460 \\
\hline
\end{tabular}

SNP: single nucleotide polymorphism; OR: odds ratio; $95 \%$ CI: $95 \%$ confidence interval; Perm: permutational $\mathrm{P}$ value for correction for multiple tests.

Table 5

Association between SNPs in DENND1B gene and asthma severity by logistic regression adjusted for sex, age, helminth infections and ancestry.

\begin{tabular}{lllll}
\hline SNP & OR & $95 \% \mathrm{CI}$ & P value & Perm \\
\hline rs4915551 (A/G) & 0.68 & $0.53-0.87$ & 0.003 & 0.002 \\
rs6691216 (C/T) & 0.62 & $0.45-0.86$ & 0.003 & 0.003 \\
rs57589685 (A/G) & 1.92 & $1.24-2.98$ & 0.003 & 0.003 \\
rs73073636 (A/G) & 1.90 & $1.28-2.83$ & 0.001 & 0.001 \\
rs16841893 (T/C) & 1.71 & $1.08-2.73$ & 0.023 & 0.024 \\
rs6694441 (C/T) & 1.67 & $1.02-2.73$ & 0.040 & 0.030 \\
rs73077640 (G/A) & 1.67 & $1.02-2.73$ & 0.040 & 0.030 \\
\hline
\end{tabular}

SNP: single nucleotide polymorphism; OR: odds ratio; 95\% CI: 95\% confidence interval; Perm: permutational $\mathrm{P}$ value for correction for multiple tests.

0.68 , CI $0.47-0.98$ ) and the allele A of rs73073636 (OR 0.71, CI $0.51-0.98$ ) were negatively associated with specific IgE serum levels for Blomia tropicalis. The allele A of rs4915551 (OR 1.23, CI 1.01-1.48) was positively associated with specific IgE serum levels for Dermatophagoides pteronyssinus.

The allele C of rs6685897 was positively associated with specific IgE
Table 6

Association between SNPs in DENND1B gene and specific IgE levels by logistic regression adjusted for sex, age, helminth infections and ancestry.

\begin{tabular}{|c|c|c|c|c|}
\hline SNP & OR & $95 \% \mathrm{CI}$ & $P$ value & Perm \\
\hline \multicolumn{5}{|c|}{ Specific IgE for Dermatophagoides pteronyssinus } \\
\hline rs6685897 (C/T) & 1.46 & $1.05-2.02$ & 0.022 & 0.027 \\
\hline rs4915551 (A/G) & 1.23 & $1.01-1.48$ & 0.030 & 0.045 \\
\hline \multicolumn{5}{|c|}{ Specific IgE for Blomia tropicalis } \\
\hline rs6685897 (C/T) & 1.38 & $1.02-1.86$ & 0.033 & 0.041 \\
\hline rs57589685 (A/G) & 0.68 & $0.47-0.98$ & 0.039 & 0.022 \\
\hline rs73073636 (A/G) & 0.71 & $0.51-0.98$ & 0.042 & 0.035 \\
\hline \multicolumn{5}{|c|}{ Specific IgE for Blatela gemanica } \\
\hline rs10801621 (G/A) & 1.53 & $1.03-2.28$ & 0.034 & 0.045 \\
\hline rs6685897 (C/T) & 1.50 & $1.02-2.20$ & 0.035 & 0.043 \\
\hline \multicolumn{5}{|c|}{ Specific IgE for Periplaneta americana } \\
\hline rs6685897 (C/T) & 1.69 & $1.10-2.61$ & 0.016 & 0.011 \\
\hline rs10801621 (G/A) & 1.72 & $1.10-2.70$ & 0.016 & 0.013 \\
\hline rs17641842 (G/T) & 1.74 & $1.09-2.77$ & 0.019 & 0.016 \\
\hline rs3902061 (G/T) & 0.52 & $0.28-0.98$ & 0.045 & 0.031 \\
\hline
\end{tabular}

SNP: single nucleotide polymorphism; OR: odds ratio; 95\% CI: $95 \%$ confidence interval; Perm: permutational $\mathrm{P}$ value for correction for multiple tests.

levels for all the allergens tested (D. pteronyssinus (OR 1.46, CI 1.05-2.02), B. tropicalis (OR 1.38, CI 1.02-1.86), B. gemanica (OR 1.50, CI 1.02-2.20) and P. americana (OR 1.69, CI 1.10-2.61)). The rs10801621 was positively associated with specific IgE serum levels for B. gemanica (OR 1.53, CI 1.03-2.28) and P. americana (OR 1.72, CI 1.10-2.70). The allele $\mathrm{G}$ of rs17641842 (OR 1.74, CI 1.09-2.77) and the allele G rs3902061 (OR 0.52, CI 0.28-0.98) were associated with specific IgE serum levels for $P$. americana, positively and negatively, respectively.

\subsection{Association of DENND1B polymorphisms with skin prick test}

The results of the analysis between the DENND1B SNPs and skin prick test are shown in Table 7. The allele C of rs6694441 and the allele G of rs73077640 were negatively associated with skin test for at least 
Table 7

Association between SNPs in DENND1B gene and skin prick test by logistic regression adjusted for sex, age, helminth infections and ancestry.

\begin{tabular}{|c|c|c|c|c|}
\hline SNP & OR & $95 \% \mathrm{CI}$ & $P$ value & Perm \\
\hline \multicolumn{5}{|c|}{ Skin prick test for at least one allergen tested } \\
\hline rs6685897 (C/T) & 1.64 & $1.21-2.2$ & 0.001 & 0.001 \\
\hline rs10801621 (G/A) & 1.62 & $1.18-2.22$ & 0.002 & 0.002 \\
\hline rs12354257 (C/T) & 1.39 & $1.12-1.72$ & 0.002 & 0.003 \\
\hline rs11799915 (G/A) & 1.31 & $1.08-1.59$ & 0.005 & 0.007 \\
\hline rs17641842 (G/T) & 1.59 & $1.13-2.23$ & 0.006 & 0.006 \\
\hline rs1998598 (G/A) & 1.32 & $1.07-1.62$ & 0.009 & 0.009 \\
\hline rs1573100 (C/T) & 1.25 & $1.04-1.51$ & 0.016 & 0.028 \\
\hline rs2884629 (G/T) & 1.29 & $1.04-1.59$ & 0.017 & 0.024 \\
\hline rs4915551 (A/G) & 1.21 & $1.02-1.44$ & 0.026 & 0.029 \\
\hline rs6694441 (C/T) & 0.63 & $0.41-0.96$ & 0.034 & 0.031 \\
\hline rs73077640 (G/A) & 0.63 & $0.41-0.96$ & 0.034 & 0.031 \\
\hline \multicolumn{5}{|c|}{ Skin prick test for Dermatophagoides pteronyssinus } \\
\hline rs6694441 (C/T) & 0.43 & $0.22-0.82$ & 0.010 & 0.009 \\
\hline rs73077640 (G/A) & 0.43 & $0.22-0.82$ & 0.010 & 0.009 \\
\hline rs1998598 (G/A) & 1.36 & $1.05-1.77$ & 0.017 & 0.019 \\
\hline rs6685897 (C/T) & 1.55 & $1.08-2.23$ & 0.017 & 0.014 \\
\hline rs12354257 (C/T) & 1.34 & $1.03-1.75$ & 0.029 & 0.026 \\
\hline rs17641524 (T/C) & 1.47 & $1.03-2.10$ & 0.033 & 0.041 \\
\hline \multicolumn{5}{|c|}{ Skin prick test for Blomia tropicalis } \\
\hline rs6685897 (C/T) & 1.83 & $1.33-2.53$ & 0.0001 & 0.0001 \\
\hline rs12354257 (C/T) & 1.48 & $1.17-1.88$ & 0.0009 & 0.0008 \\
\hline rs1998598 (G/A) & 1.42 & $1.13-1.79$ & 0.002 & 0.002 \\
\hline rs11799915 (G/A) & 1.38 & $1.12-1.71$ & 0.002 & 0.002 \\
\hline rs10801621 (G/A) & 1.65 & $1.17-2.31$ & 0.003 & 0.003 \\
\hline rs17641842 (G/T) & 1.63 & $1.14-2.34$ & 0.007 & 0.007 \\
\hline rs1573100 (C/T) & 1.31 & $1.06-1.60$ & 0.010 & 0.009 \\
\hline rs2884629 (G/T) & 1.35 & $1.07-1.70$ & 0.010 & 0.017 \\
\hline $\mathrm{rs} 17641524(\mathrm{G} / \mathrm{T})$ & 1.48 & $1.07-2.04$ & 0.015 & 0.017 \\
\hline rs4915551 (A/G) & 1.25 & $1.03-1.51$ & 0.019 & 0.028 \\
\hline rs57589685 (A/G) & 0.58 & $0.37-0.92$ & 0.020 & 0.029 \\
\hline rs6694441 (C/T) & 0.57 & $0.35-0.95$ & 0.031 & 0.029 \\
\hline rs73077640 (G/A) & 0.57 & $0.35-0.95$ & 0.031 & 0.029 \\
\hline \multicolumn{5}{|c|}{ Skin prick test for Blatela gemanica } \\
\hline rs6685897 (C/T) & 1.80 & $1.15-2.82$ & 0.009 & 0.007 \\
\hline rs10801621 (G/A) & 1.83 & $1.15-2.93$ & 0.010 & 0.007 \\
\hline rs12066841 (T/C) & 0.55 & $0.40-0.76$ & 0.0002 & 0.0001 \\
\hline \multicolumn{5}{|c|}{ Skin prick test for Periplaneta americana } \\
\hline rs6694441 (C/T) & 0.46 & $0.24-0.90$ & 0.024 & 0.030 \\
\hline rs73077640 (G/A) & 0.46 & $0.24-0.90$ & 0.024 & 0.030 \\
\hline rs6685897 (C/T) & 1.66 & $1.15-2.40$ & 0.006 & 0.005 \\
\hline rs4915551 (A/G) & 1.26 & $1.01-1.59$ & 0.039 & 0.045 \\
\hline rs12354257 (C/T) & 1.46 & $1.11-1.92$ & 0.006 & 0.007 \\
\hline rs1998598 (G/A) & 1.36 & $1.04-1.78$ & 0.021 & 0.016 \\
\hline rs10801621 (G/A) & 1.62 & $1.10-2.38$ & 0.013 & 0.011 \\
\hline rs11799915 (G/A) & 1.30 & $1.01-1.67$ & 0.037 & 0.032 \\
\hline rs2884629 (G/T) & 1.39 & $1.06-1.82$ & 0.015 & 0.014 \\
\hline rs17641842 (G/T) & 1.55 & $1.03-2.34$ & 0.034 & 0.035 \\
\hline \multicolumn{5}{|c|}{ Skin prick test for cat epithelium } \\
\hline rs12066841 (T/C) & 3.49 & $1.24-9.78$ & 0.017 & 0.012 \\
\hline \multicolumn{5}{|c|}{ Skin prick test for fungi } \\
\hline rs3902061 (G/T) & 8.48 & $2.05-35.13$ & 0.003 & 0.004 \\
\hline rs981520 (A/G) & 5.73 & $1.59-20.62$ & 0.007 & 0.011 \\
\hline rs4427436 (G/A) & 5.71 & $1.58-20.6$ & 0.007 & 0.011 \\
\hline rs2147771 (A/G) & 5.70 & $1.58-20.54$ & 0.007 & 0.011 \\
\hline rs1421396 (A/G) & 6.27 & $1.62-24.13$ & 0.007 & 0.009 \\
\hline rs1421389 (C/A) & 6.24 & $1.62-24.05$ & 0.007 & 0.009 \\
\hline
\end{tabular}

SNP: single nucleotide polymorphism; OR: odds ratio; $95 \%$ CI: 95\% confidence interval; Perm: permutational $\mathrm{P}$ value for correction for multiple tests.

one allergen tested (OR 0.63, CI 0.41-0.96), Dermatophagoides pteronyssinus (OR 0.43, CI 0.22-0.82), Blomia tropicalis (OR 0.57, CI 0.35-0.95) and Periplaneta americana (OR 0.46, CI 0.24-0.90) and the rs1998598 (OR 1.32, CI 1.07-1.62; OR 1.36, CI 1.05-1.77; OR 1.42, CI 1.13-1.79; OR 1.36, CI 1.04-1.78; respectively) and the allele C of rs12354257 (OR 1.39, CI 1.12-1.72; OR 1.34, CI 1.03-1.75; OR 1.48, CI 1.17-1.88; OR 1.46, CI 1.11-1.92; respectively) presented the inverse result, that is, positively associated with these phenotypes.

The allele A of rs4915551, allele G of rs11799915, allele G of rs17641842 and allele $\mathrm{G}$ of rs2884629 were positively associated with skin test for at least one allergen tested (OR 1.21, CI 1.02-1.44; OR 1.31, CI 1.08-1.59; OR 1.59, CI 1.13-2.23; OR 1.29, CI 1.04-1.59; respectively), B. tropicalis (OR 1.25, CI 1.03-1.51; OR 1.38, CI 1.12-1.71; OR 1.63, CI 1.14-2.34; OR 1.35, CI 1.07-1.70) and P. americana (OR 1.26, CI 1.01-1.59; OR 1.30, CI 1.01-1.67; OR 1.55, CI 1.03-2.34; OR 1.39 , CI 1.06-1.82). The allele $\mathrm{C}$ of rs6685897 was positively associated with skin test for at least one allergen tested (OR 1.64, CI 1.21-2.2), D. pteronyssinus (OR1.55, CI 1.08-2.23), B. tropicalis (OR 1.83, CI 1.33-2.53), B. gemanica (OR 1.80, CI 1.15-2.82) and P. americana (OR 1.66, CI 0.15-2.40).

The allele $\mathrm{T}$ of rs17641524 was positively associated with skin test for D. pteronyssinus (OR 1.47, CI 1.03-2.10) and B. tropicalis (OR 1.48, CI 1.07-2.04). The allele $G$ of rs10801621 positively associated with skin test for at least one allergen tested (OR 1.62, CI 1.18-2.22), $B$. tropicalis (OR 1.65, CI 1.17-2.31), B. gemanica (OR 1.83, CI 1.15-2.93) and $P$. americana (OR 1.62, CI 1.10-2.38).

The allele C of rs1573100 was positively associated with skin test for at least one allergen tested (OR 1.25, CI 1.04-1.51) and B. tropicalis (OR 1.31, CI 1.06-1.60). The allele T of rs12066841 was positively associated with skin test for cat epithelium (OR 3.49, CI 1.24-9.78) and negatively associated with skin test for $B$. gemanica (OR 0.55 , CI 0.40-0.76).

The allele A of rs57589685 (OR 0.58, CI 0.37-0.92) was associated negatively with skin test for B. tropicalis. The allele G of rs3902061, allele A of rs 981520 , allele $\mathrm{G}$ of rs4427436, allele A of rs2147771, allele A of rs1421396 and allele C of rs1421389 were positively associated with skin test for fungi (OR 8.48, CI 2.05-35.13; OR 5.73, CI 1.59-20.62; OR 5.71, CI 1.58-20.6; OR 5.70, CI 1.58-20.54; OR 6.27, CI 1.62-24.13; OR 6.24, CI 1.62-24.05; respectively).

\subsection{Association of DENND1B polymorphisms with IL-13 levels concentration on blood cell culture supernatant}

All the SNPs that presents statistical significance were positively associated with production of IL-13 by blood cell culture supernatant stimulated with mitogen (Table 8) and they are in higher linkage disequilibrium (Fig. 3B).

\subsection{GTEX in silico analysis}

The eQLT was examined to evaluate the expression of the gene depending on the genotype in tissues. GTex data that were statistically significant showed that the polymorphic allele of rs6691216 (C) (Fig. 4A) decreases the expression and the rs1998598 (G) (Fig. 3B), rs12354257 (C) (Fig. 4C), rs1573100 (C) (Fig. 4D) and rs17641524 (T) (Fig. 4E) increases the expression of DENND1B in the whole blood (Fig. 4A-E). No significant P-value was found for the eQTL analyzis for the other SNPs described in the study. (Lonsdale et al., 2013).

Table 8

Association between SNPs in DENND1B gene and IL-13 production stimulated with mitogen by logistic regression adjusted for sex, age, helminth infections and ancestry.

\begin{tabular}{lllll}
\hline SNP & OR & $95 \%$ CI & P value & Perm \\
\hline rs3902061 (G/T) & 1.78 & $1.17-2.71$ & 0.006 & 0.006 \\
rs4427436 (G/A) & 1.66 & $1.10-2.51$ & 0.014 & 0.015 \\
rs981520 (A/G) & 1.65 & $1.09-2.48$ & 0.016 & 0.019 \\
rs2147771 (A/G) & 1.64 & $1.09-2.48$ & 0.017 & 0.019 \\
rs10801621 (G/A) & 1.65 & $1.05-2.59$ & 0.027 & 0.034 \\
rs1421396 (A/G) & 1.56 & $1.04-2.32$ & 0.028 & 0.026 \\
rs1421389 (C/A) & 1.56 & $1.04-2.32$ & 0.029 & 0.027 \\
\hline
\end{tabular}

SNP: single nucleotide polymorphism; OR: odds ratio; $95 \%$ CI: $95 \%$ confidence interval; Perm: permutational $\mathrm{P}$ value for correction for multiple tests 


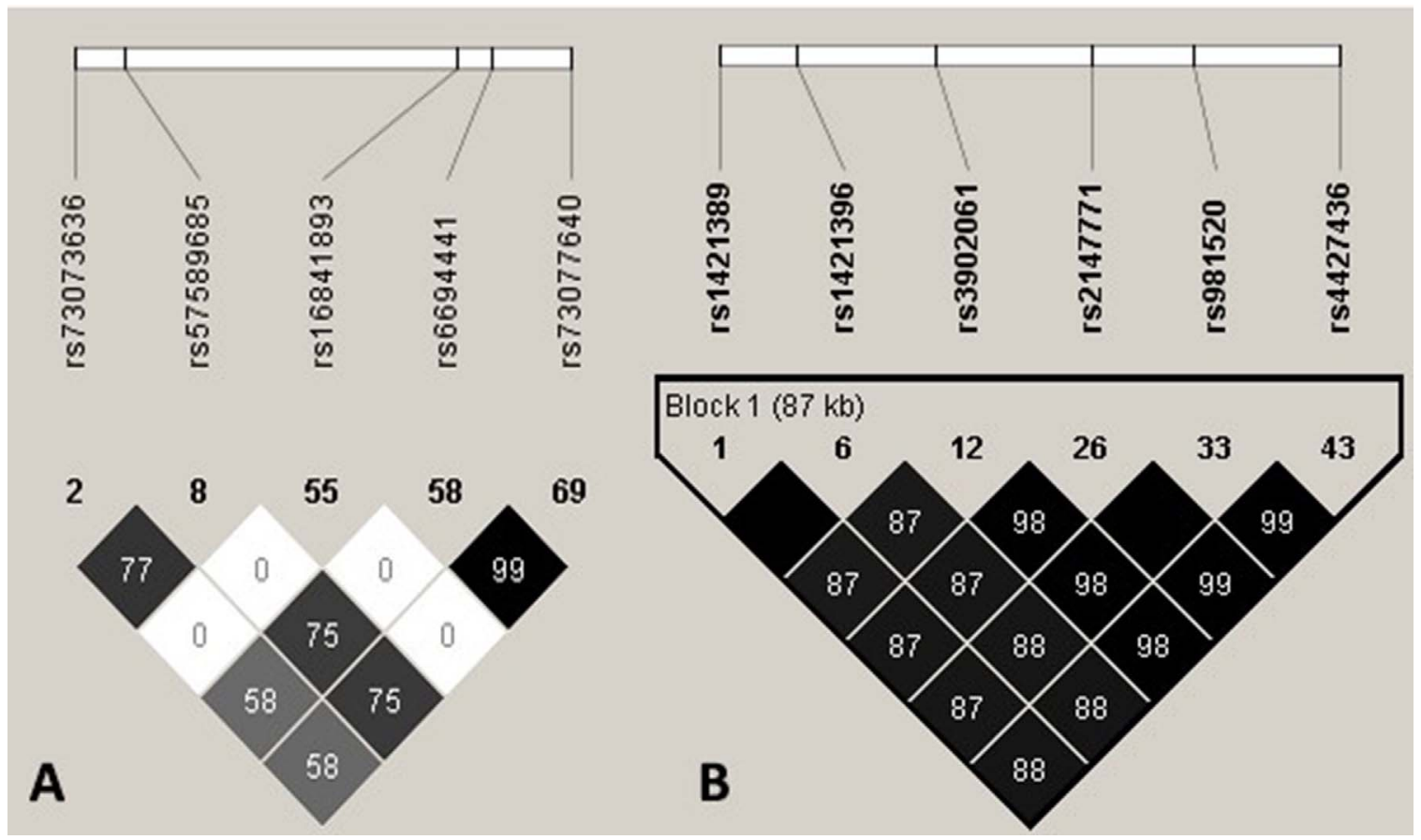

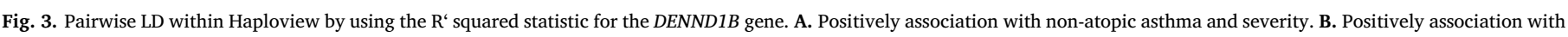
skin test for fungi and IL13 production stimulated with mitogen.

\section{Discussion}

The present study is the first to show the influence of genetic variants in DENND1B gene on asthma and atopy in a Brazilian population. We find associations of SNPs in DENND1B with asthma and atopy markers and also, for the first time, with non-atopic asthma.

In our study the rs6691216 (C) was related to asthma, acting as a protective factor for asthma, non-atopic asthma and asthma severity due to its negatively association and its score from RegulomeDB is 3a which means that this polymorphism can influence the binding of the transcription factor (TF), some motif sequences (indicates sequencespecific binding sites for proteins such as TF) and in the DNase peak, a technique to verify the influence of the SNP. The rs1421396 (A) and rs1421389 (C) were risk factors for asthma, skin test for fungi and IL-13 production stimulated with mitogen. This finding is corroborating with the Sleiman et al. (2010) in a genome wide-association study (GWAS), single nucleotide polymorphisms (SNPs) in DENND1B was related to asthma in young children. Probably these polymorphisms are interfering in the expression of DENND1B. Four SNPs reported by Sleiman et al. were found in our study. Both SNPs (rs10737692, rs12127378, rs10442656 and rs1775454) were negatively associated with skin prick test for Blatela germanica and in Sleiman's et al. study they were associated with asthma, not atopy.

Risk factors by decreasing the expression of $D E N N D 1 B$, are presumably influencing the modulation of TCR, increasing, and consequently leading to a higher production of effector cytokines leading to Th2 asthma (atopic asthma). While the protective factors act in reverse, with a controlled production of effector cytokines. Data from the GTex showed that the rs6691216 decrease the expression of DENND1B in the whole blood (Fig. 3C), in contrast with our associations results. Two subsequent studies, in multiple countries, with older children and adults did not corroborating with this finds (Ferreira et al., 2011; Moffatt, 2010). These differences may reflect the different ages of the asthmatic populations studied since genetic variants of $D E N N D 1 B$ have recently been associated with high levels of exhaled nitric oxide in healthy newborns, a phenotype associated with early-onset wheezing and a family history of allergy or allergic disorders (Chawes et al., 2015; Godar and Lambrecht, 2016) and our population is composed of children from 4 to 11 years old.

The rs1998598 (G), rs12354257 (C), rs17641524 (T), rs11799915 (G), rs10801621 (G), rs17641842 (G), rs1573100 (C) and rs2884629 (G) are risk factor for some allergen sensitizations measured by skin prick tests, indicating that these polymorphisms are more likely associated with atopy, due to the positively association with allergies markers. The rs1998598, rs12354257, rs1573100 and rs17641524 are associated positively with some allergen skin prick tests, so they are risk factors that will probably decrease the DENND1B expression but GTex data shows that these polymorphisms have higher expression of DENND1B in whole blood cell (Fig. 4B-E).

The rs6694441 (C), rs73077640 (G), rs57589685 (A), rs73073636 (A) and rs16841893 (T) were risk factors for non-atopic asthma and asthma severity and four of them were negatively associated with allergy markers, which confirms the association with non-atopic asthma and severity once these phenotype are associated with neutrophilic inflammation, are usually triggered by the presence of pathogens and pollutants and, because of that, have been shown to contribute to severe asthma and relative corticosteroid insensitivity (Brook et al., 2015). But severity is not exclusive for this asthma phenotype, since that atopic asthma also can be severe due to some allergens that causes cell damaged. The rs73073636 and rs57589685 are in linkage disequilibrium such as rs57589685 and rs6694441, and rs57589685 and rs73077640. The rs6694441 and rs73077640 are in higher linkage disequilibrium (Fig. 2A). Thus, we hypothesize that the associations of DENND1B with non-atopic asthma suggest that this gene can also be related with Th1/ Th17 cytokine production and not only specifically Th2 cytokine production as previously described for Yang et al. (2016), since this phenotype of asthma is usually triggered by Th1/Th17 immune response reaction to pathogens and pollutants.

The rs4915551 (A) was positively associated with specific IgE levels for Dermatophagoides pteronyssinu, skin test for at least one allergen tested, Blomia tropicalis and Periplaneta americana indicates that this variant is a risk factor for allergies markers and is also negatively associated with severity, corroborating the association with atopy.

The rs10801621 (G), rs17641842 (G) and rs6685897 (C) were positively associated with some skin prick test, specific serum IgE, IL-13 production by blood cell cultures stimulated with mitogen (only rs17641842) 

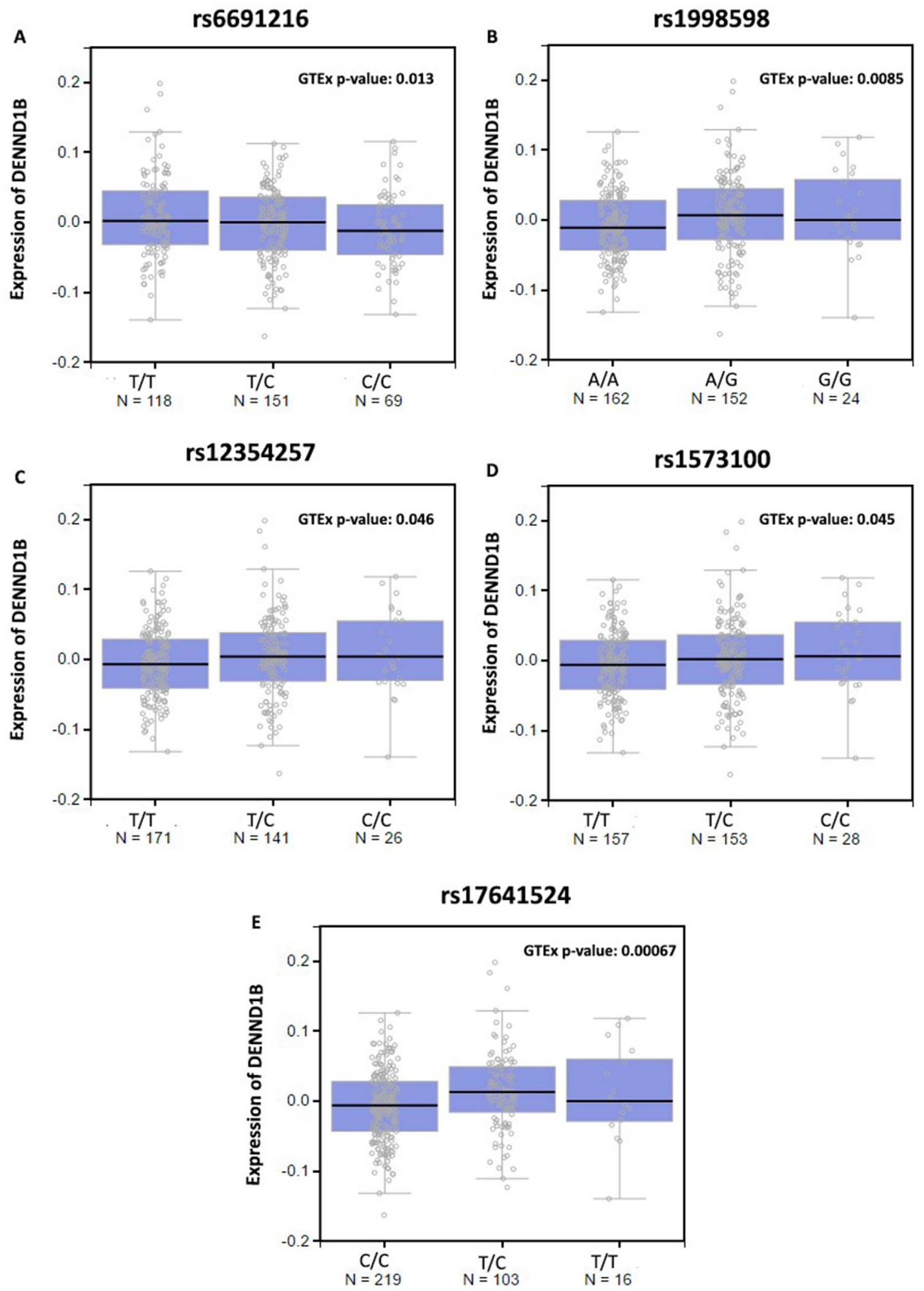

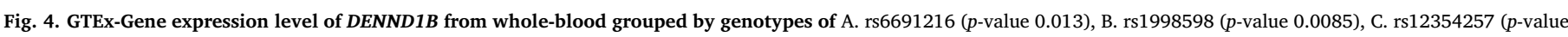
0.046 ), D. rs1573100 (p-value 0.045) and E. rs17641524 (p-value 0.00067).

and atopic asthma (only rs6685897). With this result, we can infer that they are risk factors for atopy and can increase the production of Th2 cytokines and consequently of IgE. The presence of IgE induces the mast cells degranulation and releases their cytoplasmatic granules, leading to papule formation and positivity for skin testing and also leads to an atopic asthma.
In the same way, the rs3902061 (G), rs981520 (A), rs4427436 (G) and rs2147771 (A) were positively associated with skin prick test for fungi and IL-13 production by blood cell cultures stimulate with mitogen. All the SNPs associated with skin prick test for fungi and IL-13 production are in higher or total linkage disequilibrium (Fig. 2B). 
The rs6691216, rs1998598, rs12354257, rs1573100 and rs17641524 showed opposing results in GTex. This can be explained by the fact that the project uses several populations that are different from those used in this study. Thus, ethnic components will influence the protective or risk function of SNPs, showing that is necessary to replicate this study in others populations, because the same SNP can have different outcomes depending on the population. Moreover, the majority of the polymorphisms found in this are intronic variants that can affect the mechanisms of alternative splicing and gene transcription when located in consensus sequences or sequences accentuating/inhibiting these mechanisms. That can explain the effect of the SNPs in the expression of DENND1B.

Although the study has reached its aims, there were some unavoidable limitations. First, due to limit time, it was not possible to observe the functional impact of the gene. Second, the lack of studies showing the influence of $D E N N D 1 B$ in Th1/Th17 cells makes it impossible to discuss more the association of $D E N N D 1 B$ with non-atopic asthma and the gene impact on this phenotype.

In conclusion, the polymorphisms in DENND1B gene may influence the asthma severity and the status of atopy. This was the first study to demonstrate association of the gene with non-atopic asthma, thus showing the need to identify why $D E N N D 1 B$ specifically regulates the production of Th2 effector cytokines but not the production of Th1 or Th17 effector cytokines. Also, more studies are needed to elucidate the potential role of $D E N N D 1 B$ gene on asthma and atopy, and how it could be used as a strategy to control the disease.

\section{Funding}

This work was supported by $\mathrm{CNPq}$ - National Research Council Brazil and FAPESB - State of Bahia, Brazil Research Council.

\section{Appendix A. Supplementary data}

Supplementary data associated with this article can be found, in the online version, at http://dx.doi.org/10.1016/j.molimm.2017.06.030.

\section{References}

Asher, I., Pearce, N., 2014. Global burden of asthma among children. Int. J. Tuberc. Lung Dis. 18, 1269-1278. http://dx.doi.org/10.5588/ijtld.14.0170.

Barreto, M.L., Cunha, S.S., Alcântara-neves, N., Carvalho, L.P., Cruz, Á.A., Stein, R.T., Genser, B., Cooper, P.J., Rodrigues, L.C., 2006. Risk factors and immunological pathways for asthma and other allergic diseases in children: background and methodology of a longitudinal study in a large urban center in Northeastern Brazil (Salvador-SCAALA study). BMC Pulm. Med. 10, 1-10. http://dx.doi.org/10.1186/ 1471-2466-6-15.

Barreto, M.L., Genser, B., Strina, A., Teixeira, M.G., Assis, A.M.O., Rego, R.F., Teles, C.A., Prado, M.S., Matos, S.M., Santos, D.N., dos Santos, L.A., Cairncross, S., 2007. Effect of city-wide sanitation programme on reduction in rate of childhood diarrhoea in northeast Brazil: assessment by two cohort studies. Lancet 370, 1622-1628. http:// dx.doi.org/10.1016/S0140-6736(07)61638-9.

Barreto, M.L., Ribeiro-Silva, R., de, C., Malta, D.C., Oliveira-Campos, M., Andreazzi, M.A., Cruz, A.A., 2014. Prevalência de sintomas de asma entre escolares do Brasil: pesquisa Nacional em Saúde do Escolar (PeNSE 2012). Rev. Bras. Epidemiol. 106-115. http:// dx.doi.org/10.1590/1809-4503201400050009.

Bazzi, M.D., Sultan, M.A., Tassan, N., Al Alanazi, M., Al-hajjaj, M.S., Warsy, A., 2011. Interleukin 17A and $\mathrm{F}$ and asthma in Saudi Arabia: gene polymorphisms and protein levels. J. Investig. Allergol. Clin. Immunol. 21, 551-555.

Boyle, A.P., Hong, E.L., Hariharan, M., Cheng, Y., Schaub, M.A., Kasowski, M., Karczewski, K.J., Park, J., Hitz, B.C., Weng, S., Cherry, J.M., Snyder, M., 2012. Annotation of functional variation in personal genomes using RegulomeDB. Genome Res. 22, 1790-1797. http://dx.doi.org/10.1101/gr.137323.112.

Brook, P.O., Perry, M.M., Adcock, I.M., Durham, A.L., 2015. Epigenome-modifying tools in asthma. Epigenomics 7, 1017-1032. http://dx.doi.org/10.2217/epi.15.53.

Chawes, B.L.K., Bischoff, A.L., Kreiner-Møller, E., Buchvald, F., Hakonarson, H., Bisgaard, H., 2015. DENND1B gene variants associate with elevated exhaled nitric oxide in healthy high-risk neonates. Pediatr. Pulmonol. 50, 109-117. http://dx.doi.org/10. 1002/ppul.22958.

Cherfils, J., Zeghouf, M., 2013. Regulation of small GTPases by GEFs, GAPs, and GDIs Physiol. Rev. 93, 269-309. http://dx.doi.org/10.1152/physrev.00003.2012.

Coelho, M.A. de Q., Pinho, L. De, Marques, P.Q., Silveira, M.F., Solé, D., 2016. Prevalência e fatores associados à asma em escolares de Montes Claros, MG, Brasil. Cien. Saude Colet. 21, 1207-1216. http://dx.doi.org/10.1590/1413-81232015214.04572015.

Costa, G.N.O., Dudbridge, F., Fiaccone, R.L., Silva, T.M., Conceição, J.S., Strina, A. Figueiredo, C.A., Magalhães, W.C.S., Rodrigues, M.R., Gouveia, M.H., Kehdy, F.S.G., Horimoto, A.R.V.R., Horta, B., Burchard, E.G., Pino-yanes, M., Del, B., Navarro, R., Romieu, I., Hancock, D.B., London, S., Lima-costa, M.F., Pereira, A.C., Tarazona, E., Rodrigues, L.C., Barreto, M.L., 2015. A genome-wide association study of asthma symptoms in Latin American children. BMC Genet. 16, 1-11. http://dx.doi.org/10. 1186/s12863-015-0296-7.

Ferreira, M.A.R., McRae, A.F., Medland, S.E., Nyholt, D.R., Gordon, S.D., Wright, M.J., Henders, A.K., Madden, P.A., Visscher, P.M., Wray, N.R., Heath, A.C., Montgomery, G.W., Duffy, D.L., Martin, N.G., 2011. Association between ORMDL3, IL1RL1 and a deletion on chromosome 17q21 with asthma risk in Australia. Eur. J. Hum. Genet. 19, 458-464. http://dx.doi.org/10.1038/ejhg.2010.191.

Figueiredo, C.A., Barreto, M.L., Rodrigues, L.C., Cooper, P.J., Silva, N.B., Amorim, L.D., Alcantara-Neves, N.M., 2010. Chronic intestinal helminth infections are associated with immune hyporesponsiveness and induction of a regulatory network. Infect. Immun. 78, 3160-3167. http://dx.doi.org/10.1128/IAI.01228-09.

Figueiredo, C.A., Barreto, M.L., Alcantara-Neves, N.M., Rodrigues, L.C., Cooper, P.J., 2013. Coassociations between IL10 polymorphisms, IL-10 production, helminth infection, and asthma/wheeze in an urban tropical population in Brazil. J. Allergy Clin. Immunol. 131, 1683-1690. http://dx.doi.org/10.1016/j.jaci.2012.10.043. Coassociations.

GINA, 2016. Global Strategy for Asthma Management and Prevention. Glob. Initiat. Asthma.

Godar, M., Lambrecht, B.N., 2016. Previews a new aDENNDum to genetics of childhood asthma. Cell 164, 11-13. http://dx.doi.org/10.1016/j.cell.2015.12.045.

Hawkins, G.A., Tantisira, K., Meyers, D.A., Ampleford, E.J., Moore, W.C., Klanderman, B., Liggett, S.B., Peters, S.P., Weiss, S.T., Bleecker, E.R., 2006. Sequence, haplotype, and association analysis of ADRbeta2 in a multiethnic asthma case-control study. Am. J. Respir. Crit. Care Med. 174, 1101-1109. http://dx.doi.org/10.1164/rccm.20050914050C.

Kabesch, M., Depner, M., Dahmen, I., Weiland, S.K., Vogelberg, C., Niggemann, B., Lau, S., Illig, T., Klopp, N., Wahn, U., Reinhardt, D., Nickel, R., 2007. Polymorphisms in eosinophil pathway genes, asthma and atopy. Allergy 62, 423-428. http://dx.doi. $\operatorname{org} / 10.1111 /$ j.1398-9995.2006.01300.x.

Lonsdale, J., Thomas, J., Salvatore, M., Phillips, R., Lo, E., Shad, S., Hasz, R., Walters, G., Garcia, F., Young, N., Foster, B., Moser, M., Karasik, E., Gillard, B., Ramsey, K., Sullivan, S., Bridge, J., Magazine, H., Syron, J., Fleming, J., Siminoff, L., Traino, H., Mosavel, M., Barker, L., Jewell, S., Rohrer, D., Maxim, D., Filkins, D., Harbach, P., Cortadillo, E., Berghuis, B., Turner, L., Hudson, E., Feenstra, K., Sobin, L., Robb, J., Branton, P., Korzeniewski, G., Shive, C., Tabor, D., Qi, L., Groch, K., Nampally, S., Buia, S., Zimmerman, A., Smith, A., Burges, R., Robinson, K., Valentino, K., Bradbury, D., Cosentino, M., Diaz-Mayoral, N., Kennedy, M., Engel, T., Williams, P., Erickson, K., Ardlie, K., Winckler, W., Getz, G., DeLuca, D., MacArthur, D., Kellis, M., Thomson, A., Young, T., Gelfand, E., Donovan, M., Meng, Y., Grant, G., Mash, D., Marcus, Y., Basile, M., Liu, J., Zhu, J., Tu, Z., Cox, N.J., Nicolae, D.L., Gamazon, E.R., Im, H.K., Konkashbaev, A., Pritchard, J., Stevens, M., Flutre, T., Wen, X., Dermitzakis, E.T., Lappalainen, T., Guigo, R., Monlong, J., Sammeth, M., Koller, D., Battle, A. Mostafavi, S., McCarthy, M., Rivas, M., Maller, J., Rusyn, I., Nobel, A., Wright, F., Shabalin, A., Feolo, M., Sharopova, N., et al., 2013. The Genotype-Tissue Expression (GTEx) project. Nat. Genet. 45, 580-585. http://dx.doi.org/10.1038/ng.2653.

Moffatt, M.F., 2010. A large-scale, consortium-based genomewide association study of asthma. N. Engl. J. Med. 363, 1211-1221.

Purcell, S., Neale, B., Todd-Brown, K., Thomas, L., Ferreira, M.A.R., Bender, D., Maller, J. Sklar, P., de Bakker, P.I.W., Daly, M.J., Sham, P.C., 2007. PLINK: a tool set for wholegenome association and population-based linkage analyses. Am. J. Hum. Genet. 81, 559-575. http://dx.doi.org/10.1086/519795.

Rodrigues, L.C., Newcombe, P.J., Cunha, S.S., Alcantara-Neves, N.M., Genser, B., Cruz, A.A., Simoes, S.M., Fiaccone, R., Amorim, L., Cooper, P.J., Barreto, M.L., 2008. Early infection with Trichuris trichiura and allergen skin test reactivity in later childhood. Clin. Exp. Allergy 38, 1769-1777. http://dx.doi.org/10.1111/j.1365-2222.2008 03027.x.

Simões, S., de, M., Cunha, S.S., Barreto, M.L., Cruz, Á.A., 2010. Distribution of severity of asthma in childhood. J. Pediatr. (Rio. J). 86. http://dx.doi.org/10.2223/JPED.2030.

Sleiman, P.M.A., Flory, J., Imielinski, M., Bradfield, J.P., Annaiah, K., 2010. Variants of DENND1B associated with asthma in children. N. Engl. J. Med. 362, 36-44.

Troy, N.M., Hollams, E.M., Holt, P.G., Bosco, A., 2016. Differential gene network analysis for the identification of asthma-associated therapeutic targets in allergen-specific $\mathrm{T}$ helper memory responses. BMC Med. Genomics 9, 1-17. http://dx.doi.org/10.1186/ s12920-016-0171-z.

Vercelli, D., 2008. Discovering susceptibility genes for asthma and allergy. Nat. Rev. Immunol. 8, 169-182. http://dx.doi.org/10.1038/nri2257.

Yang, C.-W., Hojer, C.D., Zhou, M., Wu, X., Wuster, A., Lee, W.P., Yaspan, B.L., Chan, A.C., 2016. Regulation of T cell receptor signaling by DENND1B in Th2Cells and allergic disease. Cell 164, 141-155. http://dx.doi.org/10.1016/j.cell.2015.11.052. 